\title{
Practical Cyber-Security Solutions for the Science DMZ*
}

\author{
Peter J. Hawrylak \\ The University of Tulsa \\ Tulsa, OK \\ peter-hawrylak@utulsa.edu \\ John Hale \\ The University of Tulsa \\ Tulsa, OK \\ john-hale@utulsa.edu
}

\author{
George Louthan \\ University of Oklahoma \\ Norman, OK \\ glouthan@ou.edu \\ Mauricio Papa \\ The University of Tulsa \\ Tulsa, OK \\ mauricio-papa@utulsa.edu
}

\begin{abstract}
The Science DMZ provides high-bandwidth and high-throughput private connectivity to or among computing resources. However, the performance comes at a cost: there is minimal security on the Science DMZ. This is by design, as conventional enterprise network security controls introduce cost, latency and bottlenecks. Data intensive applications, particularly those using geographically distributed resources, rely on the performance of the Science DMZ. With a particular lens toward multi-institution DMZs such as the OneOklahoma Friction Free Network, this paper analyzes performance and security requirements, introduces a threat model specific to the Science DMZ, and delivers recommendations for controls to mitigate these threats in the context of the identified requirements. In particular, we apply a risk-based view to the tradeoffs between performance and security, considering impacts both to the science goals of the DMZ's multiple research stakeholders and the more conventional enterprise security posture of the facilities it connects.
\end{abstract}

\section{CCS CONCEPTS}

- Security and privacy $\rightarrow$ Systems security; Network security; - Computer systems organization $\rightarrow$ Distributed architectures;

\section{KEYWORDS}

Science DMZ, Security, High-Performance Computing

\section{ACM Reference Format:}

Peter J. Hawrylak, George Louthan, John Hale, and Mauricio Papa. 2019. Practical Cyber-Security Solutions for the Science DMZ. In Practice and Experience in Advanced Research Computing (PEARC '19), July 28-August 1, 2019, Chicago, IL, USA. ACM, New York, NY, USA, 6 pages. https://doi.org/ $10.1145 / 3332186.3332213$

\footnotetext{
*Produces the permission block, and copyright information
}

Permission to make digital or hard copies of all or part of this work for personal or classroom use is granted without fee provided that copies are not made or distributed for profit or commercial advantage and that copies bear this notice and the full citation on the first page. Copyrights for components of this work owned by others than ACM must be honored. Abstracting with credit is permitted. To copy otherwise, or republish, to post on servers or to redistribute to lists, requires prior specific permission and/or a fee. Request permissions from permissions@acm.org.

PEARC '19, fuly 28-August 1, 2019, Chicago, IL, USA

(C) 2019 Association for Computing Machinery.

ACM ISBN 978-1-4503-7227-5/19/07 ..\$15.00

https://doi.org/10.1145/3332186.3332213

\section{INTRODUCTION}

High-performance computing (HPC) requires high-bandwidth and high-throughput connectivity between computing resources. One approach to achieve this is the Science DMZ. Cyber-security is an important concern for HPC resources and the data housed in them. Security requirements and HPC processing requirements are often in conflict with each other. For example, HPC requires high-bandwidth and high-throughput to ensure that messages are passed with minimal latency, often meaning no longer than it will physically take to send the packet from node A to node B. Ideally, security professionals would like to be able to inspect and verify each packet and data access, but this would not be feasible or practical.

The remainder of this paper is organized as follows. Section 2 describes the Science DMZ concept, the OneOklahoma Friction Free Network, and related work in cyber-security for HPC environments. Section 3 presents security recommendations for HPC resources. An outline for a senior level undergraduate or entrylevel graduate course covering HPC cyber-security is presented in Section 4. Section 5 presents future work and conclusions are presented in Section 6.

\section{BACKGROUND}

This section describes the Science DMZ concept which is a widely used model to deploy HPC resources on a campus network. Then the architecture and implementation of the OneOklahoma Friction Free Network which is used to link HPC resources within the state of Oklahoma is presented. The section concludes with related work and approaches to securing HPC resources is presented.

\subsection{Science DMZ}

The Science DMZ [3] is a design pattern for high-performance computing (HPC) networks, focusing on providing a network to transfer information quickly between collaborators. Delays in the transmission of information cause significant delays in scientific progress. The Science DMZ is built on three key requirements: (1) that the network is optimized to support "data-intensive science", (2) the model can scale to support more computational nodes and users, and (3) the model can evolve with technology and integrate new technologies into the Science DMZ. The Science DMZ focuses on improving performance of local networks which often have multiple operational goals. The Science DMZ is separated from the remainder of the local network to minimize the path length between it and the wide-area network and to allow it to be designed 
specifically to fit the needs for high-performance computing. The wide-area network is assumed to be designed and implemented for fast transfer of data between hosts.

According to Dart, et al. [3], the Science DMZ should be purpose built for the HPC resource and provide only what is required to support the HPC resource. This removes the need to support a widerange of operational tools (e.g., email) and this simplicity makes it easier to deploy security tools to monitor and protect the network. The security tools cannot reduce the data throughput too much but must provide protection to the network. Most security tools do not meet this "performance" requirement. The security policy needs to take the isolation of the Science DMZ from internal networks into account when defining what levels of security are required. Often, this isolation can be used to reduce the degree of certain security policies to just what is needed for the HPC applications and this reduces the performance degradation produced by the security tool. The Science DMZ solution to firewalls is to use Access Control Lists. This is possible due to the knowledge of the applications (few types of application profiles) running on the HPC equipment, and the traffic they generate.

\subsection{OneOklahoma Friction Free Network}

The OneOklahoma Friction Free Network (OFFN) [9] is a dedicated research network connecting high-performance computing resources within the state of Oklahoma. OFFN is a follow-on to the larger Oklahoma Cyberinfrastructure Initiative [10], which provides HPC access, community building, education, workforce development activities, and grant writing services to academic researchers within Oklahoma. The original OFFN implementation provided connections to the University of Oklahoma, Oklahoma State University, Langston University, and the Tandy Supercomputer Center (located in Tulsa, OK). The University of Tulsa was approved for membership after the initial deployment and has recently connected its two heterogenerous clusters to OFFN. OFFN employs the Science DMZ concept and provides users with the additional benefit of being able to experiment with Science DMZ architectures and tools. OFFN employs the perfsonar tools at each end-user site for performance monitoring.

\subsection{Cyber-Security for HPC}

An overview of security needs and possible solution approaches for HPC systems utilizing the Science DMZ network architecture is provided in [11]. HPC workloads are often similar and share several characteristics, which can be used to develop a set of signatures that can be used to detect anomalies in operation and/or usage. The regularity of HPC workloads should provide a higher success rate (lower false positive rate) in identifying attacks and allow machine learning methods to be used to classify behavior. HPC applications typically have a longer time span than IT security applications and this allows for a human to interview the user in question. The misuse of computing cycles is a major concern for HPC providers and thus they are most likely to focus their attention on their largest users. This approach provides an added benefit: that illicit use of a legitimate user's allocated cycles will expend those cycles quicker than normal or the legitimate user may detect this and inquire about this with the HPC operator. The need for scientific data provenance can be used to provide some insight into the sources of the data and can be used to identify malicious or illicit data. Protecting the integrity of the input data is a key issue for HPC applications, otherwise the results produced by those applications may be incorrect or misleading.

Many security tools utilize the command line or log files to display their output. HPC resources typically have 100 or more nodes and the command line is not the best display method to alert an administrator to anomalies. Further, monitoring systems are needed to observe the security state of the entire cluster, not just individual nodes. The command line does not provide efficient visualization of both the entire cluster and individual resources. One solution to this problem is NVisionCC [14], which is an extension of the Clumon tool to present a visual representation of the data with drill-down capabilities to the administrator. NVisionCC focuses on monitoring the processes running on the cluster and alerting the administrator when an unexpected process starts or is running. An unexpected process is likely to be related to an attack and is outside the normal usage of the cluster.

CoordiNetZ [8] is a tool to secure the network in a Science DMZ. Current software-defined-networks often used for HPC lack the capability to support dynamic data security policies. This mandates that security policies must be entered manually at all sites participating in the HPC work (HPC work may span multiple sites and institutions). They propose an intent-based policy specification framework with the capability to harmonize policies from multiple institutions and arbitrate resulting conflicts. This framework addresses three areas (1) data flow policies, (2) temporal and spatial access limitation policies, and (3) network security policies. The nature of HPC data transmissions (e.g., utilizing multiple paths to send data in parallel) make it difficult to use traditional security and network monitoring tools to detect intrusions. CoordiNetZ provides a framework to coordinate information from multiple security and monitoring sources, which is the processed and displayed to the user.

HPC resources make use of distributed exascale storage platforms that store files across a number of disk drives. This makes it difficult to deploy traditional access control security mechanisms to protect the data because it creates a bottleneck at the access control server. One solution is MaaT [6] a tool to secure high-performance computing (HPC) resources. MaaT focuses on three items (1) preventing unauthorized data access, (2) revoking access privileges, and (3) preventing common attacks. They aggregate data accesses to reduce the number of access checks required to 1 . This helps to address the issue where large datasets are distributed as a small files over a number of nodes and eliminates the need to vet the access to each individual file. Aggregating these accesses together allows a single check to be performed and covers all of the files (pieces) of the dataset. Access rights are granted in small time windows in MaaT and this simplifies the infrastructure to revoke those rights. MaaT uses the short time window to let rights that should be revoked expire and this removes the need for MaaT to communicate these revocations to the storage system. MaaT provides the capability for the user to renew their access rights.

Traditional parallel storage technologies use a meta-data server (MDS) to hold information about where each data item is stored in the object storage devices (OSDs). The MDS handles granting 
access to the different data items on the OSDs. In MaaT, every data access must first go through and be approved by the MDS. Once approved accesses can be handled directly between the user and the OSDs, this frees the MSD to service other requests. This also means that the OSDs do not have to vet accesses themselves, they trust that if the user has the credentials to access the data from the MSD that the access is acceptable.

Protecting the confidentiality and integrity of the data stored on the HPC resource is challenging. One approach is to monitor both direct and indirect information flows and block those that are deemed to be potentially unsafe. The PIGA-Cluster architecture [1] is one such tool that consists of three parts to provide some depth in securing the resource. The first part uses the standard access control mechanisms built into the operating system. The second part focuses on analysis of information flows to limit access to resources and monitor their use. The third part is the PIGA reference monitor that focuses on indirect information flows to counter more advanced attacks. PIGA uses a distributed system to check access requests (e.g., a system call) against a pre-generated list of actions that could lead to a compromise. This check is done on a dedicated, remote, node and determines if the compute node should allow the access request or not. Experiments conducted on their cluster running the linpack benchmark do not show a noticeable slowing of the operating system. However, the experiment did not include any simulated attacks on the cluster while the linpack benchmark was running.

Recent U.S. government regulations require moving HPC resources from periodic security reauthorization to a continuous monitoring approach. Malin and Van Heule [7] present findings from their investigation of implementing continuous monitoring at Las Alamos National Laboratory. Their findings show that hardware inventory is rather straightforward, but the level of granularity is important (i.e., Do you record disk drive change?). They see software configuration management to be the more difficult and a key requirement for providing continuous security monitoring. Proper implementation will keep HPC resource up to date (w.r.t. patches) and will provide administrators with a list of software so they can identify the attack surface. They recommend the use of automated tools that provide four main features to manage software configurations. First, is to use firewalls and to have a tool that checks to see if they are (1) running, (2) configured correctly, and (3) perform some tests to verify the firewall is operating correctly. Second, they recommend the use of configuration management tools and checking to (1) verify that the configuration tool is being executed periodically and (2) identify changes that have not been authorized. Third, a tool that monitors running processes on each host is recommended; unknown processes can then be flagged for investigation and trigger an alert. Fourth, the use of a tool to monitor the status of the other three security tools is recommended. Software monitoring should focus on changes to system-level software and not user-space level software because each user will install the software they need for their job in their user space and the user space is deleted (destroyed) when their job terminates.

User behavior can be a powerful tool in identifying abnormal and malicious behavior on a HPC system. Zhu, et al. [15], describe an approach to secure HPC resources by monitoring user behavior and then issuing alerts when user behavior deviates from normal behavior. This approach moves beyond securing HPC systems using only strong access control methods. Their system uses system logs to track user behavior and then applies rules based on their audit, job scheduling, and business (resource use) policies to identify abnormal behaviors. They present a solution that maps the virtual private network (VPN) account to the service node (SN) account so that the logs from the VPN session and service nodes can be correlated. The $\mathrm{SN}$ is where the user submits the job from and contains the job scheduling software, which will distribute the job to a series of compute nodes for execution. They then group events from multiple logs into a single chronological flow of events using the start and end timestamp of each event. Finally, their system maps the data flow during the user session to get a more complete picture of the user's behavior. This operation is handled on a separate node which takes the burden off of the nodes in the HPC resource.

Two-factor authentication is a powerful tool to provide increased security for authentication. Today, the most common two-factor authentication mechanism is to pair a password with a one-time code sent to a user's cell phone via text message. Texas Advanced Computing Center (TACC) recently deployed a multi-factor authentication system to enhance the security of their access control system [12]. Their implementation employs an account lockout after 20 unsuccessful login attempts to further strengthen the system. A key design requirement was that the authentication system had to be scalable and not require the user to re-enter their credentials every time they moved from one compute node to another. The use of multi-factor authentication strengthens access control and further limits the ability of a malicious or unauthorized user from gaining entry into the HPC resource.

\section{HPC SECURITY RECOMMENDATIONS}

This section presents a summary of the performance requirements for cyber-security tools for HPC resources. Following this are a series of security recommendations to meet the cyber-security requirements while ensuring acceptable computational performance.

\subsection{Performance Requirements}

Traditional HPC resources should spend the vast majority of their execution time processing jobs and not performing security checks. This mandates maximizing the use of passive security tools and passive monitoring options to secure the resources. Active security management tools will reduce the time the resource can spend on the job and the additional overhead should be minimized.

As security mechanisms are deployed, they will impact performance in two different manners. The first one is caused by hostbased monitoring components that collect and report security related information. Being deployed at the host, they are effectively using CPU cycles: CPU cycles that are not being used by the science application the HPC is supporting.

The second manner of impact is the result of communicating security related information over the network. Every time such a message is sent over the network, it reduces the amount of bandwidth that is available to the HPC application.

It is important to be able to quantify both effects in the overall operation of the HPC. Without a metric to quantify their effect, it 
will not be possible to fine tune the security apparatus. If the impact on performance is not acceptable, solutions that aggregate security related information (to reduce the size of monitoring related data), reduce the frequency of communication, or both will have to be developed.

\subsection{Security Recommendations}

Security solutions for HPC resources must balance the need for high-throughput processing of data and dedicated use of the computational resources for job execution with the needs of (1) protecting data integrity, (2) protecting data confidentiality, and (3) preventing malicious or inappropriate use of the computing resources (e.g., password cracking or Bitcoin mining). Data integrity is a core need for users who require assurances that their data will not be maliciously corrupted or altered while on the HPC resource. Data confidentiality is a core need of both the HPC administrator and user as release of the data containing personal information or other confidential data could harm the public, businesses, or government entities. Availability is the third side of the CIA (Confidentiality, Integrity, Availability) triangle and this can be limited to support stronger integrity and confidentiality safeguards. Most users access the HPC resource from the same local computer and this allows for whitelists and strong SSH keys to be shared. Limiting the user access from specific machines allows for firewalls to quickly decide which connections to accept and which to block.

Encryption of data provides a means to protect data confidentiality when data are at rest. This will prevent attacks targeting stealing data while at rest and will protect data sets when they are not being used by a job. Encrypting data during processing will add additional processing and communication overhead for the encryption/decryption operations and communication of keys that may be unacceptable for some users.

Encryption combined with a signature computed over the encrypted data and then encrypted with the data into a single package provides a means to protect data integrity. The encrypted signature provides a means to detect data tampering as the attacker will not be able to provide a proper signature for the modified data. Again, this will add processing and communication overhead to jobs, but if applied to just data at rest would only be required at the start and end of jobs.

Passive security tools appear to be the best approach inside the HPC resource. This will minimize the impact of the security tools on the main function of the resource, which is computation. Data transmission within the resource must also be maximized for both speed and throughput. Thus, comprehensive response strategies and remediation procedures are central to a HPC security policy.

Another key item is the user-interface for the security monitoring tools that administrators use. HPC resources span hundreds or thousands of devices and there is a need for both a high-level view and the ability to inspect individual systems. Security logs are often very large making it critical for the visualization tools to extract the pertinent information and display that to the user. It is critical for these dashboards to provide administrators with the ability to take action in response to perceived threats quickly. For example, a set of buttons and simple controls should be provided to allow basic actions to be taken to isolate the problem and collect necessary log files and data for further analysis. More advanced actions can be taken after the initial quarantine and starting tools to collect additional information about the attack.

\section{CURRICULUM ENHANCEMENT: CYBER-SECURITY FOR HPC}

Training of HPC practitioners is a vital need and efforts to incorporate HPC eduction into at the undergraduate structured curriculum are needed, but can be difficult due to the limited availability of technical electives and the need for students to plan course schedules several years in advance, often starting in their first year. One such approach is a minor in HPC to bridge this gap [5], however, the current structure does not include a HPC cyber-security course. Boot-camps and HPC seminars such as the one at Los Alamos National Laboratory [2] are another avenue to providing basic HPC training. Student competitions revolving around using or building HPC resources to solve a particular problem are another tool in providing the broad skill set needed by HPC professionals [4], [5]. All three of these vehicles have the added advantage of providing networking opportunities for students interested in HPC.

A dedicated course covering cyber-security for HPC environments is a needed addition to the curriculum because the additional constraints of low-latency communication significantly alter the available set of security solutions. Such a course would provide an overview of the HPC cyber-security needs and requirements, describe key design parameters for such tools, and explore tools that meet these needs and requirements.

Assuming that students in this class have a decent understanding of HPC (perhaps by adding a prerequisite to the course), we propose that this course could have the following modules: (1) computer networks, (2) cyber-security components, (3) cyber-security perimeter design and (4) cyber-security maintenance.

It is hard to understand the impact of any cyber-security solution without a basic understanding of basic computer networks. This module would cover the basics of the layering (or network stack) system typically used in any network and how messages are processed as they go up and down the stack. The module would also provide basic knowledge on metrics used to evaluate network performance and the ability to make basic latency calculations so that students understand the impact of messaging and applications on the network. This module would also cover basic communication mechanisms using TCP and UDP.

The second module will concentrate on cyber-security modules or solutions that may be deployed to secure and HPC system. The most basic solution that can be employed is that of packet filtering. Students will be exposed to the notions of static and dynamic packet filtering as well as basic recommendations for ingress and egress filtering (and their impact network performance). More advanced types of filtering, in particular stateful and proxy services will also be described. Intrusion detection system are also an important component of this module. The discussion would center on the different approaches in detecting such intrusions (anomaly-based, signaturebased, host-based, network-based). Automated countermeasures would also be discussed.

Once students have an idea of the main components they can use to secure an HPC, the third module concentrates then in the 
design of a secure perimeter. While the security requirement may be sufficient for most IT networks, this module will emphasize that, in the HPC domain, it is important that our security design minimizes its impact on the cluster. Different topologies will be covered and their impact analyzed.

The last module will concentrate on the natural life cycle of any network system. Security is a continuous cycle rather than a discrete one. Once security defenses have been deployed, it is important to be able to maintain perimeter security. A fundamental concept to be able to do this is situational awareness. This includes knowledge and operation of existing systems as well as the ability to detect rogue ones. This module should also cover incident response. NIST's SP 800-61 Rev. 2 Computer Security Incident Handling Guide provides good practices and recommendations by defining four stages of the incident response life cylce: preparation, detection $\&$ analysis, containment eradicatin \& recovery and post-incident activities.

\section{FUTURE WORK}

Artificial intelligence (AI) and machine learning approaches are potential options for analyzing log files to extract and flag abnormal behavior. Training sets for the AI tools are needed but could be developed by users from their own datasets as they will verify both the dataset and results as part of their normal research process. Often, the general workflow and possibly even the processes that will be executed are known beforehand and can be encoded into a rule-set or training set to train the AI security tool. This is generally the case with HPC jobs because of the significant time spent customizing and tuning each job for execution on the particular hardware architecture of the HPC resource. The logs from these jobs can then be archived and secured against alteration to provide a growing group of training data for a particular program. The suitability of the training data from one program to be used for another program or job is an open question.

The development of bump-in-the-wire tools are needed to provide additional monitoring and data collection. Ideally, these tools would be placed outside of the system nodes and be passive. One approach is to monitor the network for new devices or unexpected changes using such devices to passively observe network traffic and then collect this information to build network diagrams [13]. Other tools such as perfsonar can be leveraged to provide additional insight into the infrastructure. External tools can monitor this another other information collected using similar methods to maintain and monitor the current state of the infrastructure. Again, the user-interface is a key concern for such tools as critical information must be displayed in a clear and digestible fashion.

\section{CONCLUSIONS}

This paper described the current requirements for cyber-security for HPC resources. Existing approaches to securing HPC resources are surveyed in an effort to extract core requirements. The key requirements at this time are scalability of the security solution to handle hundreds or thousands of nodes, and the ability to support low-latency communication inside the HPC resource. The ScienceDMZ approach has gained much traction and supports these requirements well.
Security tools must be light-weight and provide simple userinterfaces that show both the high-level picture of the system (e.g., status of thousands of nodes) while providing the capability to obtain detailed information about individual processes and nodes. More detailed tools can then be run as the situation warrants. Tools should leverage system logs as a key means of flagging suspicious behavior and the collection of those data must be done as minimally invasive as possible.

Educational material in the cyber-security and HPC domains to train students how to protect HPC resources is needed. One possible outline for such a course to support workforce development in HPC cyber-security is presented in this paper. The authors believe this course would be well suited as a senior technical elective or Master's level graduate course and would require a prerequisite course covering the basic fundamentals of cyber-security and another prerequisite covering HPC basics. It is envisioned that this prerequisite cyber-security course would be suitable for a sophomore or junior level undergraduate course, while the HPC basics course would be a senior level undergraduate elective. It is likely that the HPC foundations course could be taken concurrently (e.g., as a co-requisite) with the proposed HPC cyber-security course.

\section{ACKNOWLEDGMENTS}

We gratefully acknowledge support from the Army Research Office, DURIP-ARO contract W911NF-15-1-0509. This material is based on work supported by the National Science Foundation under Grant No. 1531270 and also the National Science Foundation under Grant No. 1524940. Any opinions, findings, and conclusions or recommendations expressed in this material are those of the author(s) and do not necessarily reflect the views of the National Science Foundation and the Army Research Office.

\section{REFERENCES}

[1] Mathieu Blanc, Damien Gros, Jérémy Briffaut, and Christian Toinard. 2013. Mandatory Access Control with a Multi-level Reference Monitor: PIGA-cluster. In Proceedings of the First Workshop on Changing Landscapes in HPC Security (CLHS '13). ACM, New York, NY, USA, 1-8. https://doi.org/10.1145/2465808.2465809

[2] Carolyn Connor, Amanda Bonnie, Gary Grider, and Andree Jacobson. 2016. Next Generation HPC Workforce Development: The Computer System, Cluster, and Networking Summer Institute. In Proceedings of the Workshop on Education for High Performance Computing (EduHPC '16). IEEE Press, Piscataway, NJ, USA, 32-39. https://doi.org/10.1109/EduHPC.2016.10

[3] Eli Dart, Lauren Rotman, Brian Tierney, Mary Hester, and Jason Zurawski. 2013. The Science DMZ: A Network Design Pattern for Data-intensive Science. In Proceedings of the International Conference on High Performance Computing, Networking, Storage and Analysis (SC '13). ACM, New York, NY, USA, Article 85, 10 pages. https://doi.org/10.1145/2503210.2503245

[4] Stephen Lien Harrell, Hai Ah Nam, Verónica G. Vergara Larrea, Kurt Keville, and Dan Kamalic. 2015. Student Cluster Competition: A Multi-disciplinary Undergraduate HPC Educational Tool. In Proceedings of the Workshop on Education for High-Performance Computing (EduHPC '15). ACM, New York, NY, USA, Article 4, 8 pages. https://doi.org/10.1145/2831425.2831428

[5] Peter J. Hawrylak, John Hale, and Mauricio Papa. 2017. Undergraduate Educational Pathways for Developing a High-Performance Computing Workforce. In Proceedings of the Practice and Experience in Advanced Research Computing 2017 on Sustainability, Success and Impact (PEARC17). ACM, New York, NY, USA, Article 53, 4 pages. https://doi.org/10.1145/3093338.3093355

[6] Andrew W. Leung, Ethan L. Miller, and Stephanie Jones. 2007. Scalable Security for Petascale Parallel File Systems. In Proceedings of the 2007 ACM/IEEE Conference on Supercomputing (SC '07). ACM, New York, NY, USA, Article 16, 12 pages. https://doi.org/10.1145/1362622.1362644

[7] Alex Malin and Graham Van Heule. 2013. Continuous Monitoring and Cyber Security for High Performance Computing. In Proceedings of the First Workshop on Changing Landscapes in HPC Security (CLHS '13). ACM, New York, NY, USA, 9-14. https://doi.org/10.1145/2465808.2465810 
[8] Vasudevan Nagendra, Vinod Yegneswaran, and Phillip Porras. 2017. Securing Ultra-High-Bandwidth Science DMZ Networks with Coordinated Situational Awareness. In Proceedings of the 16th ACM Workshop on Hot Topics in Networks (HotNets-XVI). ACM, New York, NY, USA, 22-28. https://doi.org/10.1145/3152434. 3152460

[9] Henry Neeman, David Akin, Joshua Alexander, Dana Brunson, S. Patrick Calhoun, James Deaton, Franklin Fondjo Fotou, Brandon George, Debi Gentis, Zane Gray, Eddie Huebsch, George Louthan, Matt Runion, Joel Snow, and Brett Zimmerman. 2014. The OneOklahoma Friction Free Network: Towards a Multi-Institutional Science DMZ in an EPSCoR State. In Proceedings of the 2014 Annual Conference on Extreme Science and Engineering Discovery Environment (XSEDE '14). ACM, New York, NY, USA, Article 49, 8 pages. https://doi.org/10.1145/2616498.2616542

[10] Henry Neeman, Zane Gray, Dana Brunson, Eddie Huebsch, David Horton, James Deaton, and Debi Gentis. 2013. The Oklahoma Cyberinfrastructure Initiative In Proceedings of the Conference on Extreme Science and Engineering Discovery Environment: Gateway to Discovery (XSEDE '13). ACM, New York, NY, USA, Article 70, 6 pages. https://doi.org/10.1145/2484762.2484793

[11] Sean Peisert. 2017. Security in High-performance Computing Environments. Commun. ACM 60, 9 (Aug. 2017), 72-80. https://doi.org/10.1145/3096742

[12] W. Cyrus Proctor, Patrick Storm, Matthew R. Hanlon, and Nathaniel Mendoza 2017. Securing HPC: Development of a Low Cost, Open Source Multi-factor Authentication Infrastructure. In Proceedings of the International Conference for High Performance Computing, Networking, Storage and Analysis (SC '17). ACM New York, NY, USA, Article 37, 11 pages. https://doi.org/10.1145/3126908.3126957

[13] Bailey Smith, Whitney Caruthers, Dalton Stewart, Peter Hawrylak, and John Hale. 2016. Network Modeling for Security Analytics. In Proceedings of the 11th Annual Cyber and Information Security Research Conference (CISRC '16). ACM, New York, NY, USA, Article 22, 4 pages. https://doi.org/10.1145/2897795.2897817

[14] William Yurcik, Xin Meng, and Nadir Kiyanclar. 2004. NVisionCC: A Visualization Framework for High Performance Cluster Security. In Proceedings of the 2004 ACM Workshop on Visualization and Data Mining for Computer Security (VizSEC/DMSEC '04). ACM, New York, NY, USA, 133-137. https://doi.org/10.1145/1029208.1029230

[15] G. Zhu, Y. Zeng, and M. Guo. 2017. A Security Analysis Method for Supercomputing Users'; Behavior. In 2017 IEEE 4th International Conference on Cyber Security and Cloud Computing (CSCloud). 287-293. https://doi.org/10.1109/CSCloud.2017. 